\title{
Customer Needs and Solutions - Mission, Vision, and Strategies
}

Published online: 29 January 2014

(C) Springer Science+Business Media New York 2014

Customer Needs and Solutions (CNS) aims to be the premium outlet for scholarly work on critical issues related to customer needs (broadly defined to include the needs of both consumers and business customers) and solutions that address such needs, with the aspiration of achieving a status in management similar to that enjoyed by journals like Science or Nature. CNS contains three different types of content, Research Papers, Perspectives, and Unsolved Problems and Call for Solutions. We discuss specific strategies below in detail.

\section{Research Papers}

Conditional on the obligatory academic rigor, the journal seeks work with high upside potential for impact, and is willing to take certain risks in publishing it. The journal has adopted several unique ways to increase efficiency and democracy in the review process. Papers will be published in a timely manner and in a variety of formats (i.e., paper, online, PowerPoint, video) to reflect the changing landscape of publishing and how people currently assimilate knowledge.

\subsection{Papers Sought by CNS}

CNS seeks Research Papers with major upside potential for impact, and has high risk tolerance in doing so. The journal also does not require completeness in a paper.

- Papers with major upside potential for impact are those that could, for example, change paradigms, create substantial value for one or more stakeholders, and/or receive a very high number of citations. A paper with no upside potential will be desk rejected.

- Impact can take one or more forms including, but not limited to:
- Helping other scholars do better research;

- Helping practitioners (especially C-suite executives) create substantial value;

- Informing policy makers (and NGOs); and

- Enhancing consumer welfare.

- Seeking high return (high impact research) requires tolerating high risk. CNS is willing to do precisely this.

- High risk tolerance means we are willing to publish papers with the potential to make a substantial impact on our stakeholders, not just papers guaranteed to do so. The higher the potential upside of a paper, the higher our tolerance of this uncertainty.

- High risk tolerance means we are willing to publish controversial material, provocative results, etc., which might be proven wrong in the future, but pass the current evaluation on rigor. We may attach a "warning label" to such publications.

- High risk tolerance also means we are willing to publish papers without asking authors to "dot every $i$ and cross every $t$."

- We seek impact, not completeness. Papers otherwise considered speculative or too preliminary elsewhere are welcomed at CNS, as long as major upside potential is demonstrated.

- Examples include a new theory or framework with no data, intriguing findings with no theoretical explanation, a promising method with limited empirical validation and/ or model comparisons, a paradigm-changing idea, etc. As long as there is potential for high impact, the work will be considered.

- In short, we welcome ambitious papers that may not be fully developed because we believe such papers will accelerate the diffusion of new ideas and tools and help promote further research. 


\subsection{Supporting Evidence}

Authors are encouraged to submit supporting material to speed up the review process and to provide third-party evidence to support their work. These include, but are not limited to:

- Reviews from the following list of journals: Journal of Consumer Research, Journal of Marketing, Journal of Marketing Research, Management Science, and Marketing Science. CNS will consider manuscripts that are rejected despite merit at these journals, but fit with CNS' mission.

- Detailed supporting letters from academic experts. Authors must disclose any conflicts of interest. (e.g., coauthorship, past or current advisor/advisee, past or current colleagues at the same institution, etc.). These supporting letters may be published online in the Supplemental Materials if a paper is accepted.

- Detailed supporting letters from practitioners. Authors must disclose any conflicts of interest (e.g., financial ties, non-financial working relationships, etc.). These supporting letters will be published online in the Supplemental Materials if a paper is accepted.

- Evidence that other papers have already cited the paper, substantiating its (potential) impact.

- Other material attesting to the value of the paper. (Authors may check with the EIC before submission.)

\subsection{Why Should You Publish Your Best Research Papers in CNS?}

- A paper published in $C N S$ will acquire the prestige analogous to papers at Science or Nature in the eyes of anyone who cares about customers and solutions. Your work will be associated with other papers characterized by high relevance and impact.

- You can publish your brilliant work that is considered too radical or insufficiently developed by existing journals; such papers are welcomed and sought by $C N S$.

- You will enjoy a very efficient review and publication process with no additional rewriting required before submission. Low revision effort will be required if we like the work and we will reuse existing reviews from other journals listed under Supporting Evidence.

- You can provide alternate evidence that supports the value of your work, fostering the democratic nature of the publication process. This reduces the chance of a paper being rejected due to the idiosyncratic preferences of one or two particular reviewers.

- You will get maximum impact from your work. In addition to publishing a traditional paper journal, CNS will disseminate the work in a way that harnesses the power of new media technology in the digital age (i.e., online Supplementary Materials, video, audio, PowerPoint, Twitter, etc.). CNS will actively promote the work to stakeholders (i.e., firms, policy makers, NGOs, media, academics) and will aim to stimulate follow-up research.

\section{Perspectives}

This section contains invited (non-peer reviewed) articles, and aims to serve two purposes. First, it provides a conduit for communication between academics and stakeholders (i.e., business practitioners, policy makers, NGOs, etc.). Second, it provides a venue for thought leaders and experts to share their opinions. The EIC, in consultation with members of the editorial team and scholars in general, will invite people to contribute to this section. Please contact EIC directly if you want to suggest a topic and/or an individual (including yourself) for a possible article for Perspectives.

\subsection{Purpose}

- Provide a conduit of communication between academics and stakeholders.

- Provide an outlet for thought leaders.

- Increase the probability of impact on practice (and the likelihood that practitioners will read journal content).

2.2 Type

- Interviews of CEOs (or key C-suite members) and other senior stakeholders

- Interviews of Deans

- Commentaries from thought leaders (academics, practitioners) on the direction of the field, practices, methods, etc.

- Panel discussions by academics in a specific domain or by senior executives with similar responsibilities.

\subsection{Format}

- Questions will be solicited from the editorial board members before each interview/panel discussion.

- Interviews and panel discussion, if possible, will be either audio or video recorded and will be made available online after editing.

- During these interviews and panel discussions, feedback will be solicited on specific research papers published in CNS. 


\section{Unsolved Problems and Call for Solutions}

This section publishes unsolved problems that are important and related to topics covered by the journal. The goal is to help shape the research agenda and to be proactive in encouraging certain research. Unsolved problems (with data necessary to potentially solve a problem, where appropriate) can be submitted by anyone for peer review. We publish the most important unsolved problems, and extend invitations for papers that can provide satisfactory solutions to these problems.

\subsection{Purpose}

- Create a core set of important problems that can take the guesswork out of research ("Am I working on a problem that our discipline thinks is important?"). Unlike research priorities set by various entities (e.g., research centers and institutes), the parameters of these problems are precisely defined to allow unambiguous solutions to be created (see examples below). Also, unlike existing outsourcing R\&D sites where participants may compete to solve problems posted by a firm, the unsolved problems published by $C N S$ should be fundamentally interesting to the discipline, and solutions should be valuable to a broad audience.

- Encourage scholars to work on these problems by promising publication of best solutions to these problems.

- Increase our impact by providing "free consulting" to firms that want to solve their problems (as long as they are valuable to a broader audience).

- Make more datasets available for academics.

- Broadly, guide the research agenda in marketing.

\subsection{Submission (Author, Format, Data Availability)}

- Anyone (faculty, doctoral students, companies, NGOs, consultants, etc.) can submit any important problems that have not been solved (or those where existing solutions need improvement). The author of a problem may also pledge to provide a cash reward to whomever creates a solution (i.e., when the author represents a firm).

- Problems must be well-defined (not vague).

- The author of a submitted problem should either make appropriate data available for anyone who is interested in solving it, or the data should be publicly available and/or relatively easy to collect. Authors should notify the EIC how they will make data available and how academics can access the data (e.g., Will data be public? Will certain forms need to be signed in order to access the data? Can academics use the data for other purposes?)

\subsection{Examples}

- Netflix forecasting challenge (if one is willing to make such data available).

- A better metric than Net Promoter Score (NPS), or customer satisfaction index.

- More generally, for example, one could provide data along with the best available model in the discipline, and ask others to come up with a new estimation model that increases the prediction rate to a certain level.

- Or, one could state that it is thought that $\mathrm{A}, \mathrm{B}$, or $\mathrm{C}$ is causing $\mathrm{X}$ and ask others to come up with a clever way to identify the real causal relationship (which may even be D).

\subsection{Review Process}

- Submitted problems will be reviewed for importance and data availability.

- The EIC will handle the review, with the help of one or more editorial team members as needed (the SE, AE or a reviewer).

\subsection{Call for Solutions}

- Anyone who is interested in identifying solutions to an unsolved problem published in the journal should consult with the EIC before starting their work.

- The EIC will appoint one SE to handle all submissions of solutions for a given unsolved problem published in the journal.

- Satisfactory solutions will be published as Research Papers in the journal, possibly jointly (re)written by all scholars who have independently provided good solutions.

3.6 Why Should One Submit an Unsolved Problem to CNS (and Make Your Data Available)?

- It is a scholarly contribution to our field to identify an important and unsolved problem. Problems are peer reviewed, and a person who identifies a problem will be forever associated with the solution. Any future award given to a paper that is based on an unsolved problem may be shared between the author of the problem and the author of the solution if appropriate.

- We expect that authors either do not know how or do not have time to solve the problems they submit. 
- For firms, this presents an opportunity to obtain free consulting from the best minds in the academic world. It also provides promotional opportunities for firms.

- For NGOs and policy makers, this is an opportunity to solve urgent problems without spending grant money.
As a journal designed to be risk tolerant in the pursuit of high impact work, CNS itself will also be evolving as it experiments with bold new strategies to best achieve its mission. We welcome not only your best scholarly work, but also your comradeship as a fellow traveler in this journey. 